\title{
Transient thyrotoxicosis following external beam radiotherapy to the neck
}

\author{
R. Mallik, S. Ponnampalam, J. Ahlquist \\ Southend University Hospital
}

\section{Introduction:}

Hypothyroidism is a recognised late complication of radiotherapy to the neck.

However, radiation-induced thyrotoxicosis is not generally considered, and may not be diagnosed.

We report a case of transient thyrotoxicosis following radiotherapy to the neck.

\section{Case Description:}

- A 71-year-old man with supraglottic squamous cell carcinoma was treated with 65Gy radiotherapy, delivered by volumetric arc therapy in 38 fractions.

- During radiotherapy he became confused. 3 days after completing radiotherapy a blood test revealed thyrotoxicosis: TSH $<0.01 \mathrm{mU} / \mathrm{L}$, fT4 $36.5 \mathrm{pmol} / \mathrm{L}$, fT3 $10.1 \mathrm{pmol} / \mathrm{L}$.

- On examination he had a fine tremor, increased sweating and tenderness over the anterior neck. There were no signs of thyroid eye disease.

- No past history or family history of thyroid disease.

- Radiation-induced thyroiditis was suspected, and thionamide treatment was not given.

- Four days later fT4 had fallen to $24.8 \mathrm{pmol} / \mathrm{L}$. TPO antibody and TSH-receptor antibody were negative.

- After six weeks thyroid function had returned to normal, TSH $3.05 \mathrm{mU} / \mathrm{L}, \mathrm{fT} 412.5 \mathrm{pmol} / \mathrm{L}$. A thyroid uptake scan was planned but was not performed.

\section{Discussion:}

- It is well recognised that radiotherapy to the neck may lead to late hypothyroidism.

- However, radiation-induced thyrotoxicosis may not be generally recognised.

- There have been isolated reports of thyrotoxicosis due to Graves' disease developing years after neck radiotherapy.

- In this case the early development of transient thyrotoxicosis indicates the diagnosis of radiationassociated thyroiditis.

\section{Learning Points:}

In an oncology setting the clinical features of tachycardia and sweating may be mistaken for acute illness and thyrotoxicosis may not be diagnosed.

We suggest that if thyrotoxicosis develops soon after neck radiotherapy, thyroiditis should be suspected and treatment with thionamides should not be given.

Thyroid status should be monitored closely for resolution and progression to hypothyroidism.

Awareness of this condition by endocrinologists and oncologists will help avoid inappropriate treatment and will ensure appropriate monitoring and follow-up. 